\title{
FPGA-Based Reconfigurable Measurement Instruments with Functionality Defined by User
}

\author{
Guo-Ruey Tsai and Min-Chuan Lin \\ Department of Electronics Engineering, Kun-Shan University of Technology, Taiwan
}

Received 2 October 2004; Revised 5 March 2005; Accepted 25 May 2005

\begin{abstract}
Using the field-programmable gate array (FPGA) with embedded software-core processor and/or digital signal processor cores, we are able to construct a hardware kernel for measurement instruments, which can fit common electronic measurement and test requirements. We call this approach the software-defined instrumentation (SDI). By properly configuring, we have used the hardware kernel to implement an $n$-channel arbitrary waveform generator with various add-on functions, a wideband and precise network analyzer, a high-speed signal digitizer, and a real-time sweep spectrum analyzer. With adaptively reconfiguring the hardware kernel, SDI concept can easily respond to the rapidly changing user-application-specified needs in measurement and test markets.
\end{abstract}

Copyright ( 2006 Hindawi Publishing Corporation. All rights reserved.

\section{INTRODUCTION}

As the power of FPGA increases $[1,2]$, we find ourselves with the ability to design, simulate, analyze, and even emulate the more complex devices with application-specified embedded processor and/or digital signal processor cores. From the viewpoint of SDI concept [3], the process of measurement has been reduced only to signal excitation, captures, conditioning, processing, and output display as illustrated in Figure 1 [4]. Figure 2 illustrates that the traditional instrumentation technique depends on digital signal processor, microprocessor unit, virtual instruments, application-specified integrated circuit (ASIC), or FPGA, which are in charge of the responsibility of signal conditioning and signal processing.

The instrument market is fragmented because instruments are specialized in hardware to serve thousands of slightly divergent test applications. In fact, the traditional classification of measurement instruments (such as voltmeter, frequency counter, function generator, oscilloscope, signal analyzer, etc.) has become blurred, and to some extent can be replaced with a single set of reconfigurable hardware, called hardware kernel. The hardware kernel can be reconfigured by software to implement a specified measurement instrument. With such a software-defined architecture concept applied to the circuit level, we have two advantages. First, it can dramatically reduce the number of hardware components in all mixed-signal designs. This then possibly means a much smaller chip size for system-on-chip implementation. Second, it can provide automatic adjustment or compensation for circuit component variations due to temperature dependence, aging, manufacturing tolerances, and so forth.

Current high-performance FPGA is richly equipped with built-in on-chip SRAM, which includes block RAM and distributed RAM. Therefore, either logic circuits using tablelookup algorithm or embedded processor in system-onchip application could utilize the on-chip SRAM to improve the speed degrading due to external chips' interconnection and then enhance the entire system performance. Under a single-hardware-core architecture, all the implemented instruments are meant only to adjust the instrumental functions in software way and apply them to their specified application fields. In Section 2, we illustrate the system architecture of the proposed hardware kernel first. In Section 3, we introduce five kinds of possible instrument design algorithms by SDI philosophy: multichannel arbitrary function generator, DC transfer curve tracer, transient response analyzer, steady-state network analyzer, and real-time spectrum analyzer. In Sections 4, 5, 6, and 7, we will demonstrate the practical implementation of four signal processing devices: an $n$-channel arbitrary waveform generator with various add-on functions [5], a wideband and precise phase detector [6], high-speed signal sampler by multiple-path algorithm [7], and all-digital real-time spectrum analyzer [8] In Section 8, a flexible re-configuration methodology of this SDI system is presented. Finally, we have come into a conclusion. 


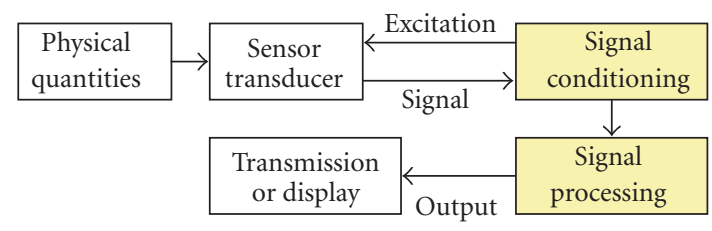

FIgUre 1: Measurements technique by SDI.

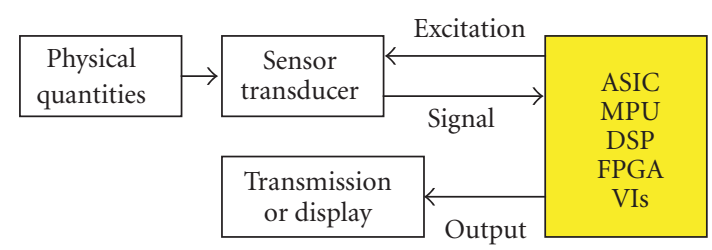

Figure 2: Instrumentation technique.

\section{HARDWARE KERNEL FOR THE RECONFIGURABLE INSTRUMENTS}

Figure 3 illustrates the proposed hardware kernel architecture. Besides FPGA, we need other ASIC chips to process analog signals. In order to measure time, frequency, and phase responses of the device under test (DUT), we need the following function modules: digital-to-analog converter, waveform amplifier, analog-to-digital converter, waveform sharpener, phase detector, hardware peak/trough detector, and human input devices (HIDs).

The original stimulus signal generated by FPGA is in digital form. For analog exciting signal requirement, it must be converted by digital-to-analog converter, filtered and shaped by low-pass-filter, and amplified or attenuated by amplifier or DC offset. The amplitude of the exciting signal can be adjusted through automatic gain control which is achieved by FPGA-generating programmable gain-adjustment (PGA) signal.

We also need signal capture and digitization modules. The output signals from DUT can be digital or analog. The latter needs to be captured and digitized by analog-to-digital converter. To meet the input signal limitations to analog-todigital converter, the signal gain of output analog signals still needs to be controlled by the PGA signal which is generated by FPGA.

We need to detect the inevitable phase drift between input and output signals from DUT. By waveform sharpening circuit, we can transform the periodical analog signal into square wave. The phase difference can be drawn out from the duty cycle of the square wave. The duty cycle is calculated by the FPGA or ASIC chip. The process of phase detection is shown as Figure 4.

To calculate sine wave excitation and response amplification factor, we need peak extraction circuit to detect two peak-to-peak values and get the quotient between them. From the data array, the embedded processor in FPGA can take out the peak values (maximum or minimum) for further processing.
All human interface devices (HID) for manipulation and test data presentation are basic interfaces for each instrument. The proposed hardware kernel includes the following HIDs: push wheel switch, led, text LCD, graphic display STN/LCD or color TFT/LCD, keyboard, touch panel, even oscilloscope signal driver.

The flash RAM can be used to store sine, Log, or other mathematic function lookup tables for exciting signal generation, and ease fast data operations.

This system can be operated in on-line mode and the personal computer (PC) can control and communicate with it. Without the PC, this system is also a stand-alone device offline operated by panel components and displayed to liquid crystal display (LCD). We have designed the panel controller and LCD controller using the embedded processor.

For on-line operations, we can design a PC development platform with powerful graphic unit interface (GUI) and mathematic functions package, which can be supported from Matlab or LabVIEW. On the other hand, the hardware kernel should have some on-line operation interfaces, such as USB, SPI, or UART to communicate with the PC.

\section{RECONFIGURABLE INSTRUMENTS}

With the complete hardware kernel architecture, we can configure FPGA to match the necessary function specifications for various measurement environments and requirements. Here we introduce five types of SDI design algorithms for specified applications.

\subsection{Arbitrary waveform generator}

Utilizing direct digital synthesizer (DDS) [2, 9-11] algorithm, we can generate any periodic function with arbitrary frequency, amplitude, and waveform. As illustrated in Figure 5, the function waveforms are preloaded into flash RAM, and will be directly loaded into the built-in RAM in FPGA when powered up. The waveform frequency can be set up to half the system clock. Using 32-bit phase accumulator can achieve a frequency resolution of $0.02 \mathrm{~Hz}$. The embedded 8 - bit processor in FPGA is in charge of the control of HIDs and setting calculation of frequency and amplitude. Arranged as Figure 6, we reorganize the DDS data processing path and generate two channels of FM, PM, FSK, or PSK signals. Section 4 will describe an $n$-channel arbitrary waveform generator with various add-on functions in detail.

\subsection{DC transfer function analyzer}

We use a transfer function analyzer to analyze the transfer function between input and output signals, and we can observe the linearity characteristics of the measured sensor/transducer. The input/output transfer curve measurement is essential for characterizing DUTs with electronic circuits. Arranging the FPGA software design flow as in Figure 7, we can configure the proposed hardware kernel into a DC transfer function analyzer. 


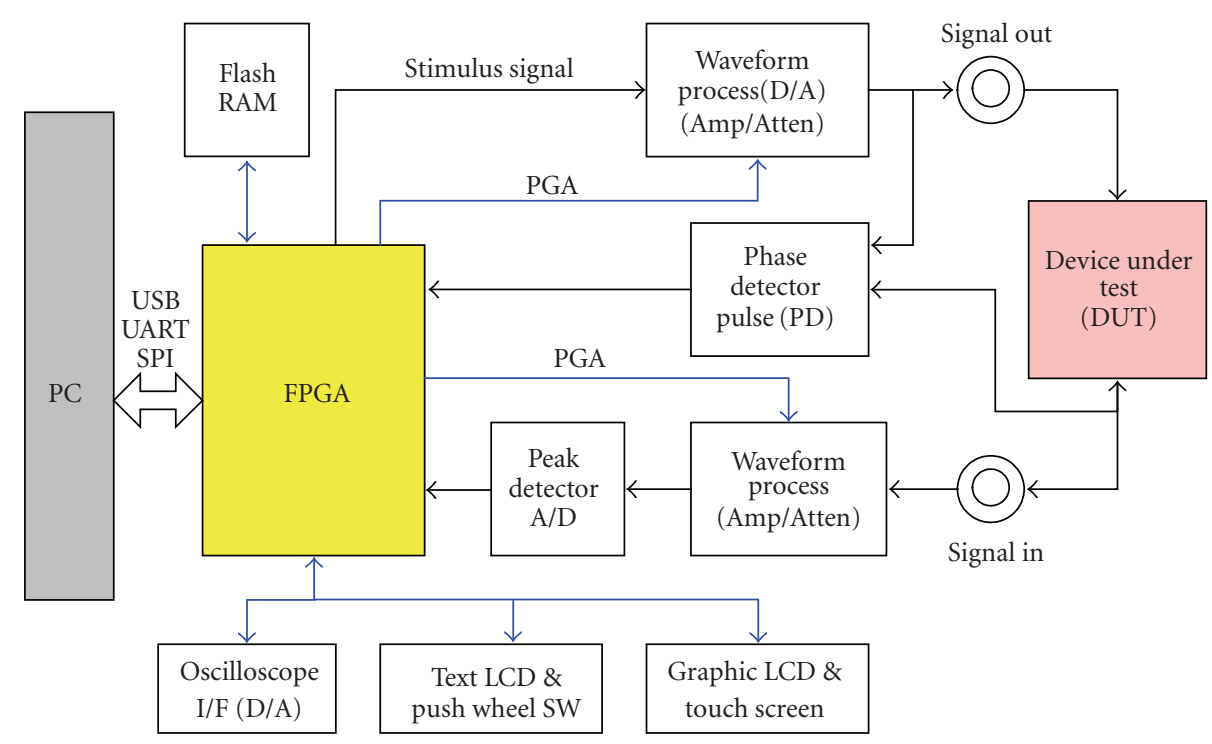

FIgURE 3: Hardware kernel for the reconfigurable instruments.

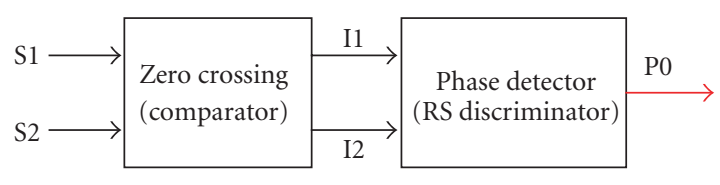

FIgure 4: Phase detection

\subsection{Steady-state network analyzer}

For the hardware kernel as in Figure 3, when using DDS technique to generate sweep sine wave, and retrieving the phase and peak response of the DUT, we can collect the tabular data of the system frequency response. The frequency response spectrum can be constructed by calculating the tabular data in logarithm by the embedded processor and put into display on the color graphic LCD. We also can upload the data array to a PC for further processing. Section 5 will describe a wideband and precise network analyzer based on FPGA in detail.

\subsection{Transient-state analyzer}

When the proposed arbitrary function generator generates synchronized periodic signal as the required exciting signal which is fed into the DUT, we will get the time response output which needs further transient analysis, as shown in Figure 8. To overcome the lower sampling rate of the analog-to-digital converter, a multipass algorithm is proposed. Section 6 will describe a multipass algorithm digitizer based on FPGA in detail.

We can have $n$-time the effective sampling rate. When the exciting signal is designed and presented as a periodically variable duty-cycle square wave, a step response analyzer is built up. If an FFT algorithm is built into the FPGA, the hardware kernel will be configured as a software-based spectrum analyzer.

\subsection{Real-time spectrum analyzer}

Figure 9 illustrates a real-time sweep spectrum analyzer using a fixed IF filter and a sweeping local oscillator (LO). The mixer output contains the input signal, the LO signal, the sum and difference between these two signals, and various other frequency components. If we know the LO frequency exactly, then by sending these frequency components through a narrow IF filter, we can identify both the amplitude and the frequency of the unknown input signal. Whenever any of these components falls within the IF filter bandwidth, an AC voltage, which is related to the input signal's amplitude, is produced. This AC voltage is converted to a DC voltage by an envelope detector, and the result is displayed on the $y$-axis of the screen.

By HDL coding or schematic entry, we can implement the mixer, narrow IF filter, envelope detector, voltage-control oscillator (VCO), and other processing algorithms into the same FPGA chip. Section 7 will describe an FPGA-based design of real-time sweep spectrum analyzer in detail. The next section describes the developed $n$-channel arbitrary waveform generator with various add-on functions.

\section{4. n-CHANNEL ARBITRARY WAVEFORM GENERATOR WITH VARIOUS ADD-ON FUNCTIONS [5]}

\subsection{DDS waveform generator}

DDS is the most popular technique to synthesize AC incentive signals for instrumentation, measurement, and digital communications. Generating synthesized waveforms by DDS technique has the following benefits: high frequency resolution, precise frequency control, and low complexity. Figure 10 shows the simplified DDS block diagram [9].

Utilizing conventional table-lookup algorithm for DDS, we need not generate both sine and cosine functions and can realize desired functions with smaller memory table size. We 


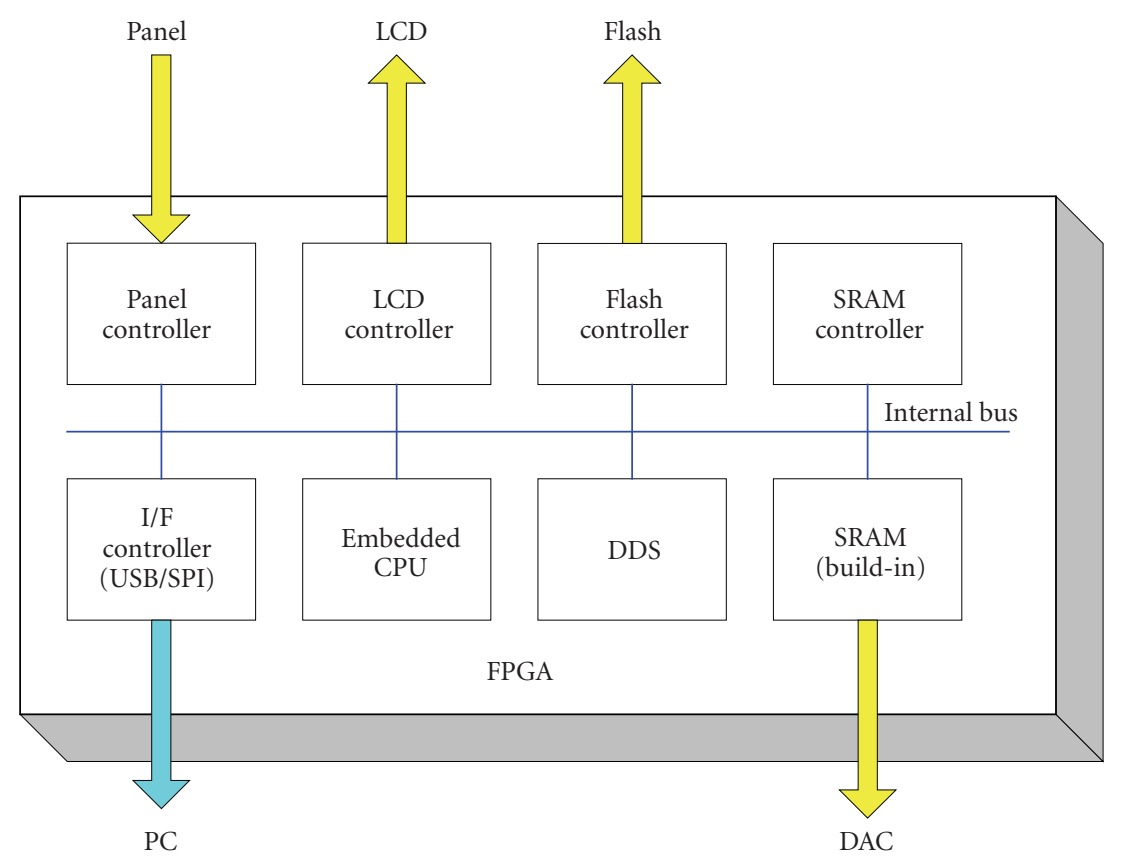

FIGURE 5: System architecture for arbitrary $n$-channel function generator.

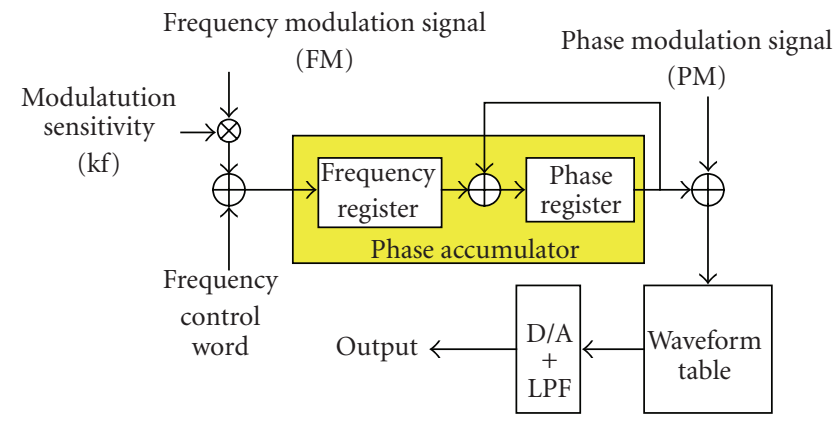

FIgURE 6: On-chip FM/PM modulation.

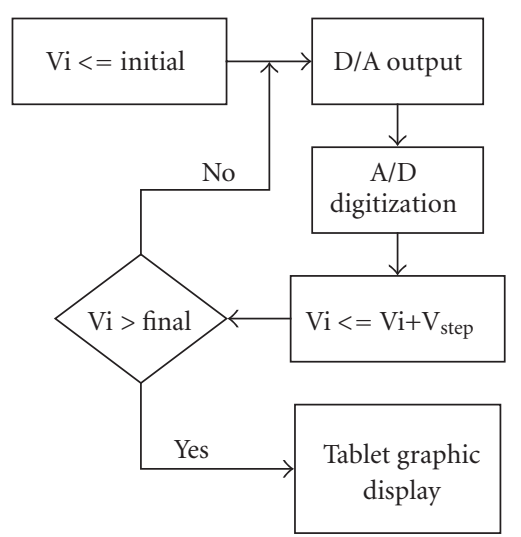

Figure 7: DC transfer function measurement. can implement all the necessary digital logic circuits and the lookup memory in the same FPGA chip so that a better performance can be achieved by avoiding interchip connections $[3,10]$. To generate the required analog signals, commercial digital-to-analog converters (DAC) can be adopted. Lowpass filters (LPF) are required to filter out high frequency noise.

The entire instrument system includes a PC platform, USB controller, FPGA waveform synthesizer, and DAC/LPF output buffer, as shown in Figure 11. The PC is the system development platform, responsible for arbitrary function waveform editing, previewing, encoding, lookup data downloading, and the coding and decoding of USB commands. The multiple operation windows and GUI application programs are coded by Visual Basic language.

The USB controller will deal with the messages interchange between PC platform and FPGA chip. The Cypress EZ-USB controller is utilized to communicate the PC with the FPGA. It provides some DLL files, which can be called and linked by Visual Basic, Visual C languages, and/or LabVIEW programs in the PC platform, and simplifies the design for both messages interchange and transmission control of GUI windows. Besides the parallel interface, we can also use the SPI or $\mathrm{I}^{2} \mathrm{C}$ techniques to communicate between USB controller and FPGA to save the pins resource of the FPGA. By the plug-n-play property of USB interface, a PC can be used to develop many AWG instruments simultaneously.

\subsection{FPGA realization}

The FPGA is used to synthesize the specified function waveform. We adopt Xilinx Spartan II XC2S200PQ208 which 


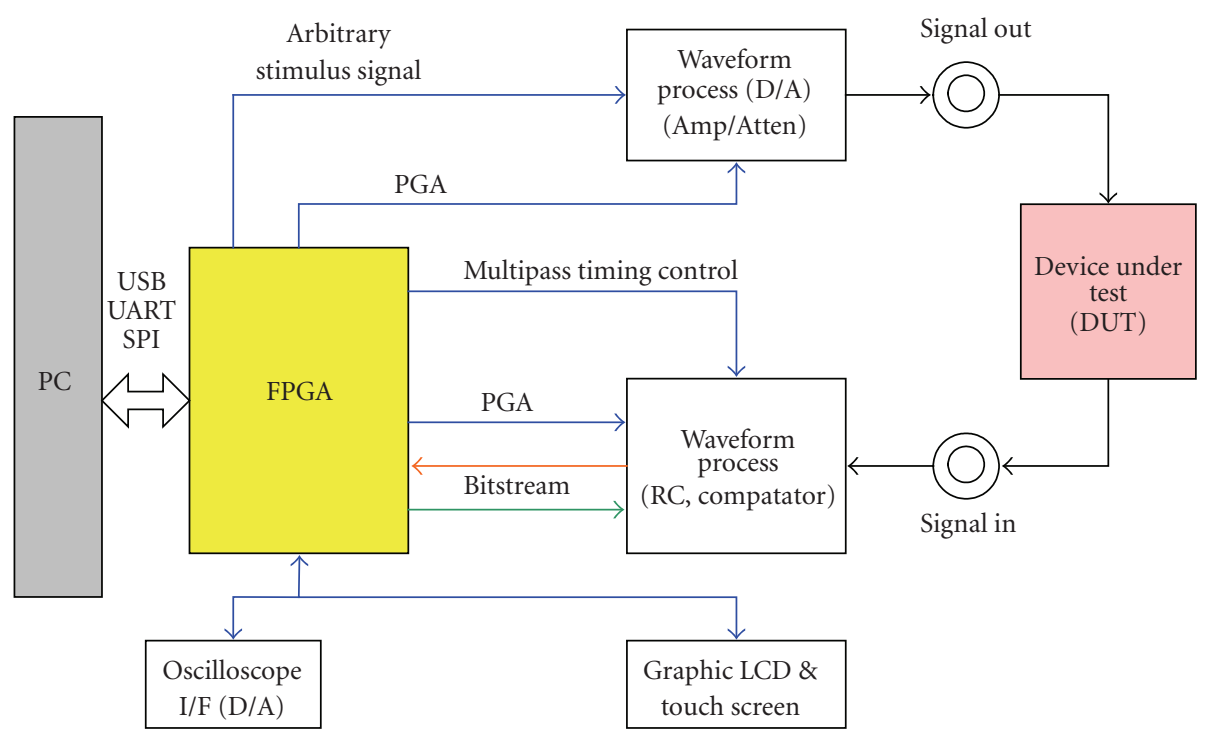

Figure 8: Transient state analyzer.

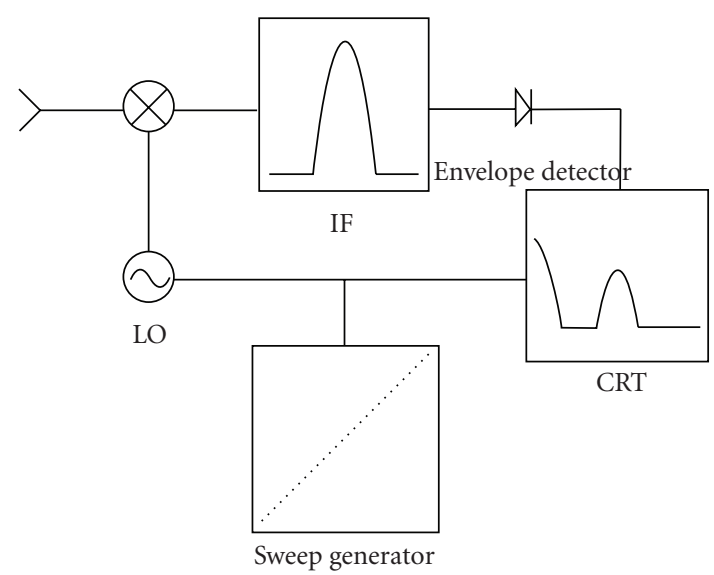

Figure 9: Real-time sweep spectrum analyzer.

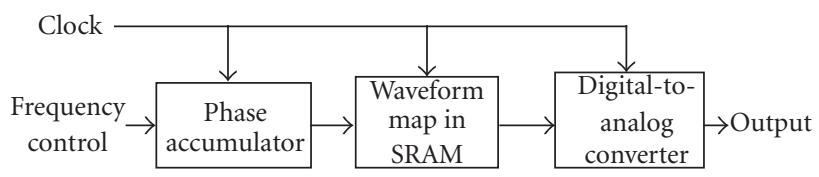

FIgURE 10: Simplified block diagram of the direct digital synthesizer.

affords on-chip true single-port blocked synchronous RAM. The total available memory size is 56 kbit. The FPGA chip is configured into four main parts: hand-shaking controller between USB and FPGA, SRAM (block RAM), SRAM controller, and remaining control logic. The SRAM is utilized for both built-in and downloadable lookup tables. The builtin lookup table can also be reserved for specified waveforms output directly or built-in self-test purpose for the instrument itself. Together with $n$ DACs, we can reconfigure the SRAM capacity into $n$ parts for $n$-channel analog signal outputs. In this case, we have up to 56 channel outputs using 1 kbit per channel. On the PC platform, you can download each channel waveform one by one. Adopting $50 \mathrm{MHz}$ clock frequency and 32-bit phase accumulator word length, we have a $0.01164 \mathrm{~Hz}$ frequency resolution.

\subsection{Function performance}

After integrating all the interfacing software, firmware, and hardware, the instrument can afford typical fundamental waveforms output, such as sinusoidal, square, and triangle functions. We can also edit any mathematical equations in the edit window and output their waveforms. Typical modulated waveforms, such as AM, FM, and others, can be edited and stored into the waveform banks in advance. You can choose anyone you like from the waveform banks and freely output to any desired channel. The instrument also provides piecewise linear function output with multiple data points periodically. Choosing FPGA with larger on-chip SRAM capacity for lookup table usage, we can flexibly expand the output channel numbers or improve the waveform resolution. With the programmability in the PC development platform, we can output the waveforms to individual channels independently, or generate a mixed waveform, which is a linear combination among several other channels.

Furthermore, we can produce a series of $n$-channel waveforms, which show some group-related functions for special application purposes. Figure 12 shows the typical output results of the instrument. Figures 13(a) and 13(b) show two clear frequency spectrums for $1 \mathrm{kHz}$ sinusoidal waveforms produced by this instrument and Agilent 33120A signal generator individually. We have nearly the same waveform quality. 


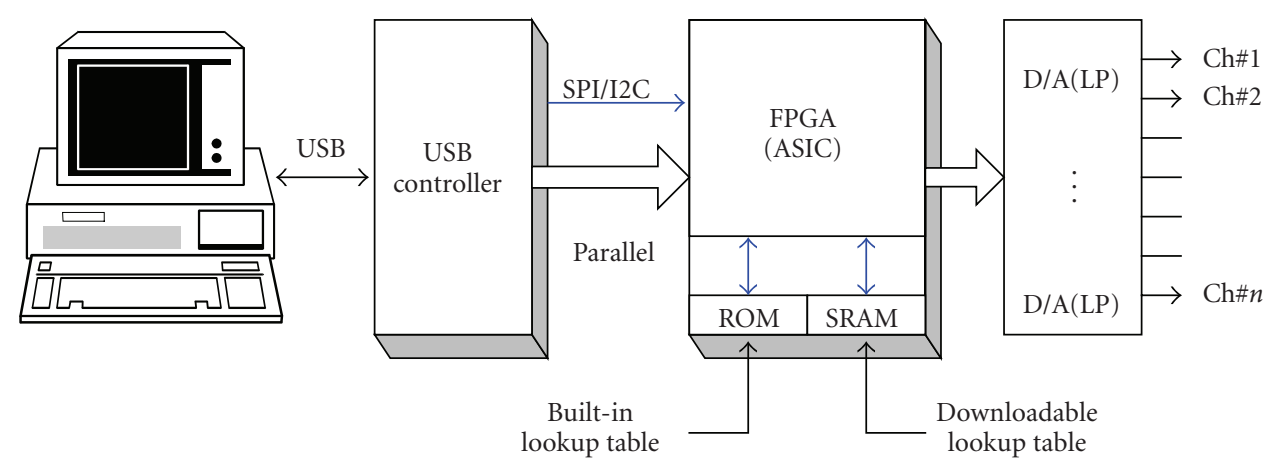

FIgURE 11: The system architecture of the $n$-channel arbitrary waveform generator.

\subsection{Add-on function}

The instrument also provides an algorithm for more advanced custom-made waveforms generation. Taking a custom-made FSK signal generator as the example, you can input or edit a modulation bitstream on the PC platform, and download it to the SRAM in FPGA chip. Processed by a preset FSK control code, we can combine both the bitstream channel and sine wave channel to generate the desired FSK signal. Figure 14 shows the custom-made signal generation flow.

The algorithm receives the data from both sine wave and piecewise linear generators, and respectively decides the frequency for " 1 " and " 0 " which can control the accumulator phase increment generator and construct the designed FSK signal. AM-ASK control code can be coded to generate the designed AM-ASK signal by the same approach. With the reserved input port, the external FSK control code IP can directly be fed into and generate the designed FSK signal waveform by the stand-alone instrument.

\section{WIDEBAND AND PRECISE PHASE DETECTOR BASED ON FPGA WITH EMBEDDED PROCESSOR [6]}

We can use the multiplier phase detector [4] or logarithmic amplifiers to implement a phase detector. But these two approaches are both mixed-mode type and unsuitable for system on-chip (SoC) design which is in all-digital type. There exist several digital design methodologies for phase detector, such as EXOR phase detector, JK flip-flop phase detector, phase-frequency detector, Nyquist-rate phase detector, zerocrossing phase detector, and Hilbert-transform phase detector $[12,13]$. But they are all only suitable for some specified narrowband frequency range. To detect phase in another frequency range, we must modify phase detection and calculation circuits to meet the necessary requirements, and then be able to get the precise value of phase difference.

\subsection{All-digital phase detector}

The proposed phase detector is an all-digital approach to measure the phase difference of two signals with the same frequency. Gathering the signal frequency by control circuit in FPGA and calculating data by the embedded processor in FPGA, we can adaptively adjust the sampling clock, which is used to measure the pulse period. This phase detector automatically detects and adjusts the sampling clock without any circuits' modification.

Figure 15 demonstrates the proposed all-digital adaptive algorithm for phase detection, including incoming signal's frequency recovery circuit, sampling clock generator, and alldigital phase detectors. The period of sampling clock, $\mathrm{T}_{s}$, is the function of both income signal frequency $\left(f_{s}\right)$ and phase resolution $(\Delta p)$. And the phase value, $P_{d}$, is the function of both sampling period $\left(T_{s}\right)$ and the pulse duration of phase difference $\left(\Delta P_{t}\right)$ :

$$
\begin{aligned}
T_{s} & =f\left(f_{s}, \Delta p\right), \\
P_{d} & =f\left(T_{s}, \Delta P_{t}\right) .
\end{aligned}
$$

From (1), we must get the incoming signal frequency and desired phase resolution at first, and then put them into the programmable fractional- $\mathrm{N}$ frequency synthesizer. Finally, we have the sampling clock required for phase detection. Combining the sampling clock and a specially designed counter algorithm, we can get a phase difference value with 8 to 12 bits from (2).

\subsection{FPGA realization}

We design the whole system with our proposed phase measurement method for specified network analyzer applications and implement it by FPGA, as illustrated in Figure 5. From the operation point of view, we have USB interface for PC on-line operation, and embedded processor for standalone off-line control. The DDS-powered function generator is used for sweep stimulus signal generator and sampling clock generator. The all-digital phase lock loop (PLL) with programmable divide-by- $\mathrm{N}$ module is used for the phase detection of uncontrollable random input signals. And an LCD display controller is also included for stand-alone use display.

Utilizing DDS algorithm, we can generate any periodic function waveform with arbitrary frequency, amplitude, and waveform. The embedded 8-bit processor in FPGA is in 


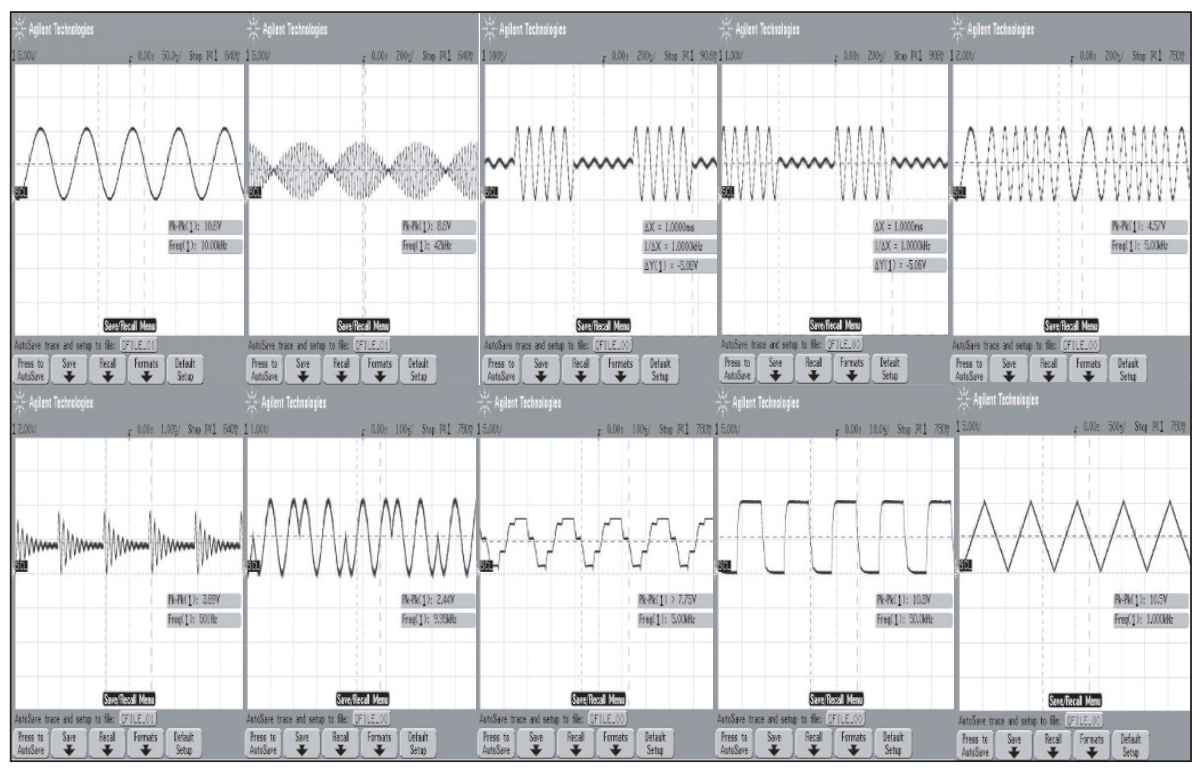

FIgUre 12: Typical output waveforms displayed by Agilent 54622D oscilloscope.

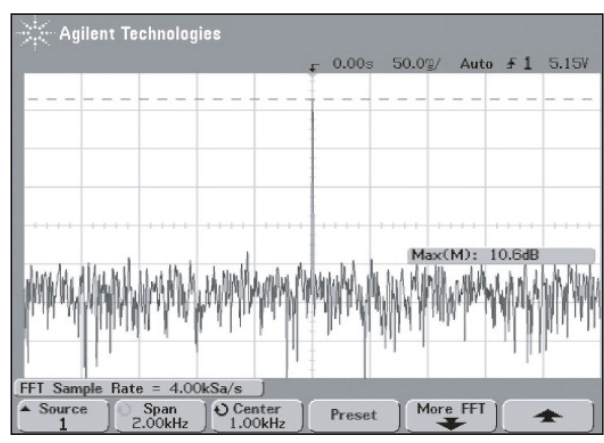

(a)

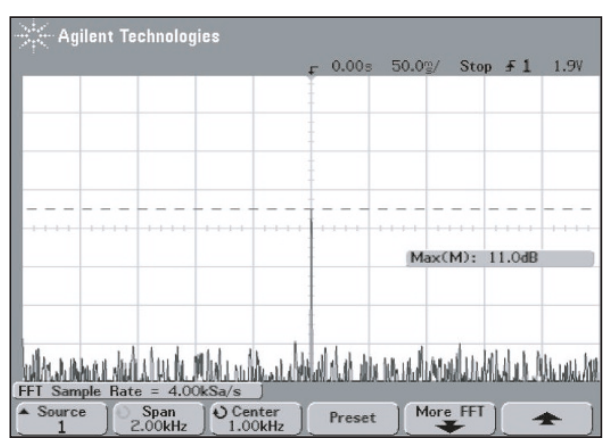

(b)

FIGURE 13: Displayed $1 \mathrm{kHz}$ sinusoidal spectrum comparison between (a) the reconfigurable instrument and (b) Agilent $33120 \mathrm{~A}$ signal generator. The spectrums are displayed by Agilent 54622D.

charge of the control of USB communication and setting calculation of frequency, amplitude, and sampling clock.

When the input signal of DUT is not generated by the system function generator, but comes from other uncontrollable signal source, we use both the all-digital phase locked loop and fractional- $\mathrm{N}$ frequency synthesizer [14], as illustrated in Figure 16, to recover input signal's frequency and generate required sampling clock. To meet the requirement of precise resolution, the programmable scale-factor- $\mathrm{N}$ divider must generate the counting clock required for calculating the duration of phase difference. Constituted by counters, the all-digital phase detector can output 8- to 12-bit digital signals for phase calculation according to the requirement of phase resolution and the successive processing circuits.

\subsection{Performance analysis}

Table 1 lists the comparison of measured phase differences of RC circuit from 10 to $10 \mathrm{kHz}$ measured by Agilent 54621A oscilloscope and our proposed method, respectively. From the results, it is apparent that our proposed method is precise enough, and the measurement frequency range is relatively wide without specified parameter adjustment and further special logic circuits.

When we adopt automatic sweep stimulus signal generator, which is controlled by a PC, to generate the input signal, and collect and analyze the data by LabVIEW program, we have the amplitude and phase response as shown in Figure 17. It is evident that the measurement is rather precise within a wide frequency range. It supports that our proposed method is suitable for all-digital SoC system realization.

\section{HIGH-SPEED SIGNAL SAMPLER BY MULTIPLE-PATH ALGORITHM [7]}

To implement an A/D converter into a pure digital chip, a delta-sigma D/A algorithm must be built in to work together with SAR, flash, or other types of A/D algorithms. The slow conversion rate of delta-sigma D/A algorithm is the main 


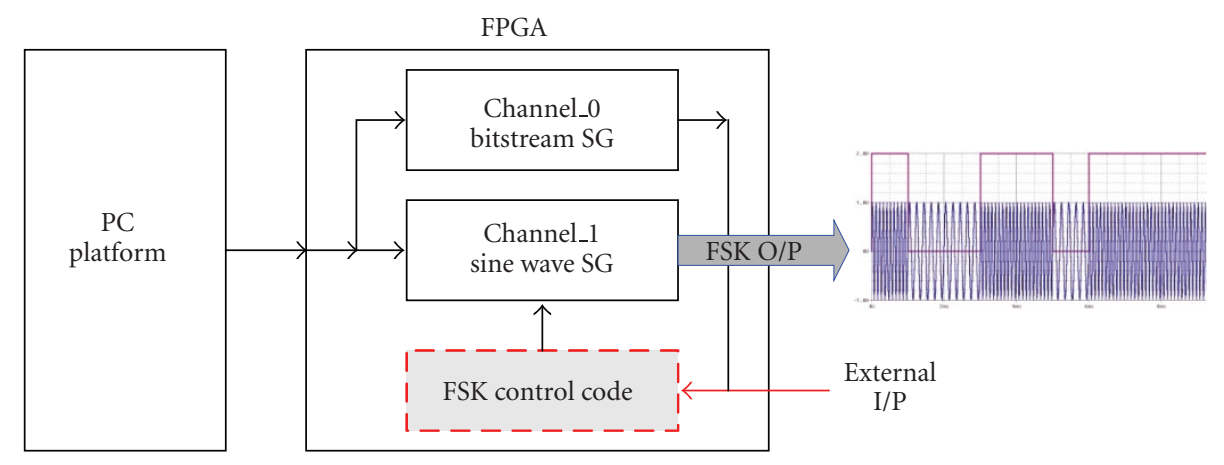

FIgURE 14: The customer-made FSK signal generation flow.

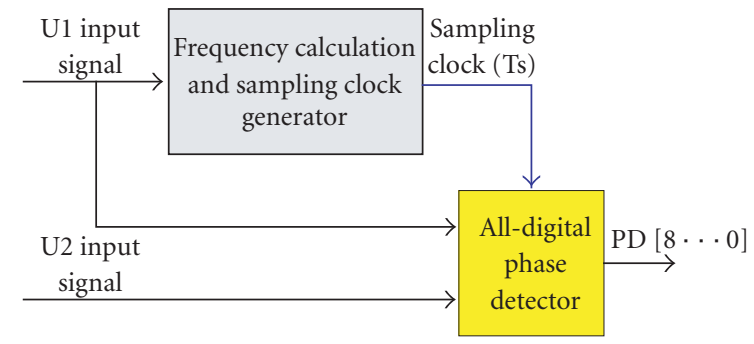

FIGURE 15: All-digital adaptive algorithm for phase detection.

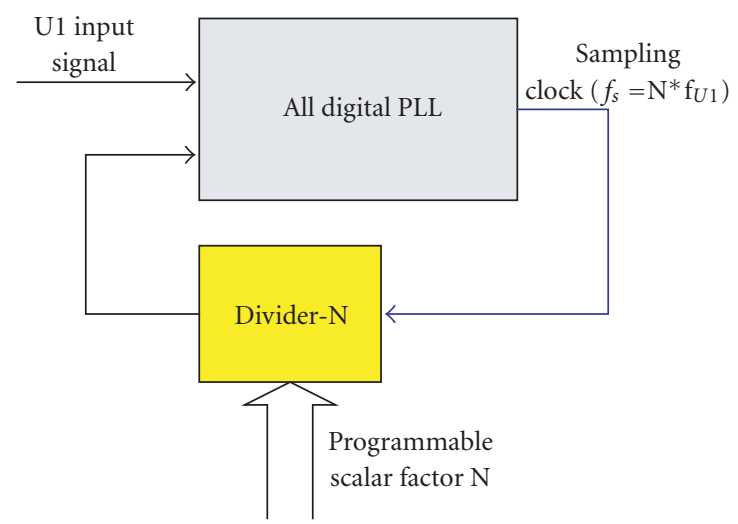

FIGURE 16: All-digital PLL fractional-N frequency synthesizer.

limitation to the whole A/D converter for capturing highbandwidth periodic signals.

\subsection{Multipass method}

According to the sampling theory, we need a sampling rate two times larger than the greatest signal frequency so that the original signal can be reconstructed after it was sampled. The higher the sampling rate is, the more complete the signal reconstruction is. For a periodic signal, if we periodically sample the signal whose frequency can be larger or even less than the signal frequency with the available sampling device, we can get a set of sampled signal values. Then, we take a fix
TABLE 1: Comparison of the measured phase differences of RC circuit.

\begin{tabular}{c|cc}
\hline \multirow{2}{*}{ Frequency $(\mathrm{Hz})$} & \multicolumn{2}{|c}{ Phase measured (degree) } \\
\cline { 2 - 3 } & Agilent 54621A & Proposed method \\
\hline 10 & 0.00 & 0 \\
50 & 0.00 & 0 \\
100 & -4.32 & -4 \\
500 & -16.20 & -16 \\
$1 \mathrm{~K}$ & -31.68 & -32 \\
$2.5 \mathrm{~K}$ & -52.20 & -51 \\
$5 \mathrm{~K}$ & -64.80 & -64 \\
$10 \mathrm{~K}$ & -72.00 & -71 \\
\hline
\end{tabular}

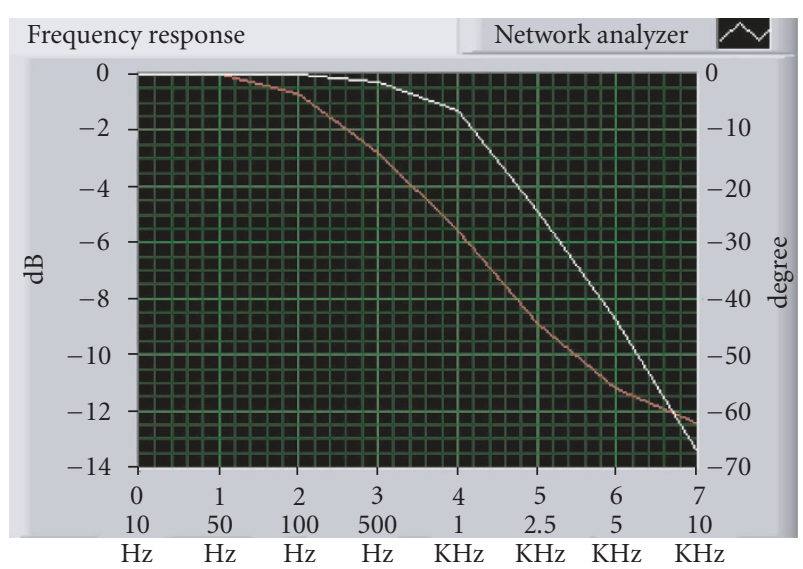

FIGURE 17: Automatic sweep RC-circuit frequency response by our system.

time shift $t_{\text {shift }}$, calculated as follows:

$$
\Delta t_{\text {shift }}=\frac{\text { sampling }- \text { time }}{n},
$$

where $n$ is an integer. We can repeatedly and synchronously get $n$ sets of sampled signal values, and store them into the embedded memory in FPGA. Figure 18 demonstrates the proposed multipath sampling algorithm. 


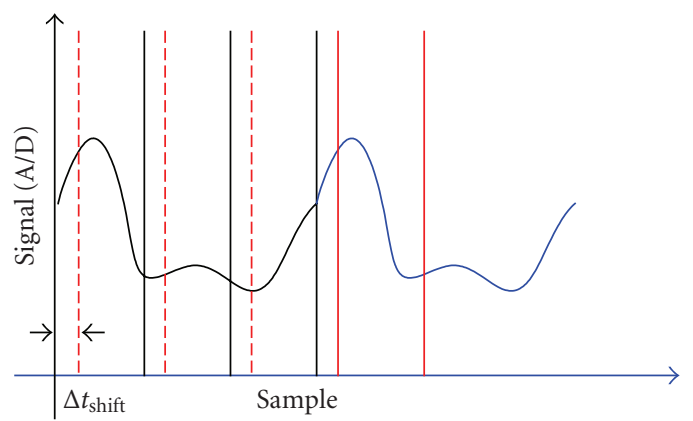

- Pass 1 sample -- - Pass 2 sample

FIGURE 18: Multipass under-sampling algorithm.

By this way, the apparent sampling rate is $n$ times higher than the real-time sampling rate. If $n$ is large enough, we can overcome the problem of the low real-time sampling rate and get more satisfactory signal reconstruction.

\subsection{FPGA realization}

We use an FPGA chip, an RC circuit, an LF398 sample/hold chip, and an LM319 comparator chip to implement the multipass method, as shown in Figure 19. The built-in DDS arbitrary waveform generator is used to generate the desired periodical signal waveform whose data stream is stored in the waveform data table. The external R-2R circuit is used to convert the digital data streams into continuous analog signal. To verify the captured signal quality, we directly bypass the output signal into the sample/hold chip so that we can compare the original and the captured signals. Through the comparator chip, the compared result between the output signal from sample/hold chip and the RC circuit output signal is fed into the SAR A/D converter. The delta-sigma D/A converter outputs the bitstreams to the RC circuit, and then the charged output signal is fed into the comparator chip. The internal DDS module provides the necessary synchronous signal for repeated sampling. If the tested signal comes from other signal source, we need the all-digital phase lock loop (ADPLL) module (Figure 16) for signal synchronization and timing control. The memory controller is in charge of the storage and transfer of the digital data streams. The TDC signal extractor is used for signal reconstruction. The interface circuit can be used for PC communication and data transfer controller.

\subsection{System performance}

In this demonstration, we use $50 \mathrm{MHz}$ of system clock rate. We use the DDS module to generate a sine wave with frequency of $100 \mathrm{kHz}$ as the stimulus signal. From the step response of RC circuit, as shown in Figure 20, we have the $151 \mu$ s steady state rise time and $136 \mu$ s fall time.
By estimation, the minimum converting time of the SAR $\mathrm{ADC}$ is about $160 \mu \mathrm{s}$. In other words, the real-time maximum signal capture sampling speed is about $780 \mathrm{~Hz}$ for 8 quantum bits. According to the calculation formula of the realtime sampling rate of the delta-sigma D/A converter, shown as follows, we have the real-time sampling rate $760 \mathrm{~Hz}$ for ADC_bit $=8$ and $F=15$ :

$$
\mathrm{SR}=\frac{50 \mathrm{MHz}}{2^{\text {(ADC_bit }+1)} \times(F+1) \times \text { ADC_bit }}
$$

Figure 21 shows the comparison of original input $100 \mathrm{kHz}$ sine wave and the captured signal by the proposed signal capturer. The captured signal has 256 sampled points per period. We can find that the signal reconstruction is rather satisfactory. We have demonstrated an ultrahighspeed signal capturer based on a single FPGA chip. The multipath algorithm has enhanced the sampling rate from a $760 \mathrm{~Hz}$ real-time sampling rate up to a $25.6 \mathrm{MHz}$ apparent sampling rate. For further applications, we can use this design to measure the transient response of the device under test.

\section{ALL-DIGITAL REAL-TIME SPECTRUM ANALYZER [8]}

A spectrum analyzer is used to analyze the frequency components in signals under test. By mathematical calculation, the traditional fast Fourier transform (FFT) can transform the time-domain waveform to frequency-domain waveform of the signal and become the key technique of the spectrum analyzer. The sweeping frequency technique combined with digital technique is also used to implement spectrum analysis [15]. In general, the analysis accuracy of FFT technique is basically worse than that of the sweeping frequency technique equipped with digital intermediate-frequency filter. From the viewpoint of dynamic range decaying effect, the FFT technique is also worse. For smaller frequency range, the processing speed of FFT technique is better, but worse for wider frequency range. In the form of digital intelligent property (IP), which can be mapped to a single FPGA chip, we design a real-time sweep spectrum analyzer. The system function block diagram of real-time sweep spectrum analyzer is shown in Figure 22.

\subsection{Theory of operation}

The mixer is used to combine the signal under test and the sweeping signals generated by the local oscillator (LO). The mixer is a multiplier circuit, so the output is an amplitudemodulated signal. According to the formula of triangular algebra, we have the signals with sum frequency and difference frequency, as shown in the following expression:

$$
\begin{aligned}
& \sin \left(2 \pi f_{0} t\right) \times \sin \left(2 \pi f_{\text {in }}\right) \\
& \quad=\frac{1}{2} \cos 2 \pi\left(f_{0}-f_{\text {in }}\right) t-\frac{1}{2} \cos 2 \pi\left(f_{0}+f_{\text {in }}\right) t
\end{aligned}
$$

where, $f_{0}$ is the frequency of LO, $f_{\text {in }}$ is the input signal fre- 


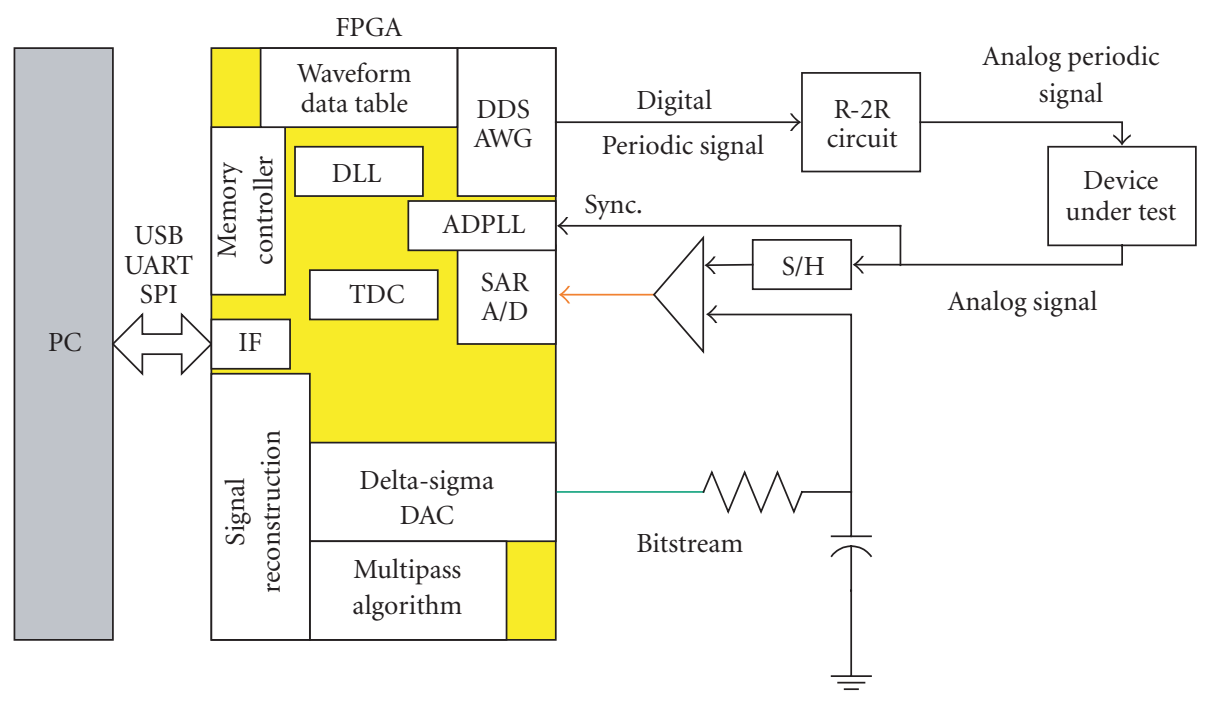

FIGURE 19: FPGA-based all digital signal capturer.

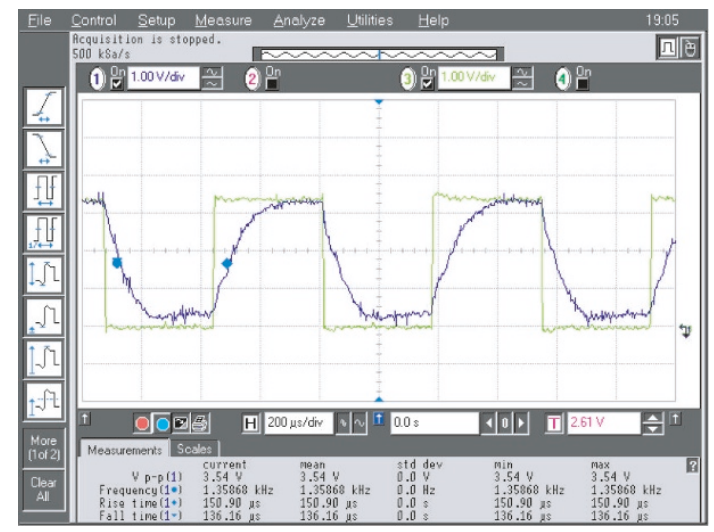

FIgURe 20: The step response of delta-sigma D/A converter.

quency. The central frequency of the finite-impulse-response (FIR) bandpass filter $\left(f_{\mathrm{IF}}\right)$ is

$$
f_{\mathrm{IF}}=f_{0}+f_{\text {in }} .
$$

When the AM signal is passed to the FIR filter, the signal with frequency band meeting the pass band of the filter can pass through. The following peak detector can get the passed signal's amplitude, which will be shown in the X-Y mode of oscilloscope.

If we fix the LO frequency, then we must adjust the central frequency of FIR filter adaptively to properly detect all the frequency components of the signal under test. The adaptive central frequency must satisfy (6).

It seems that the IF filter with adaptive central frequency is not practical from the viewpoints of cost, accuracy, and speed. In contrast, keeping the central frequency of filter fixed, and linearly (or logarithmically) sweeping the LO frequency, we have the following relation:

$$
f_{0}=f_{\mathrm{IF}}-f_{\text {in }},
$$

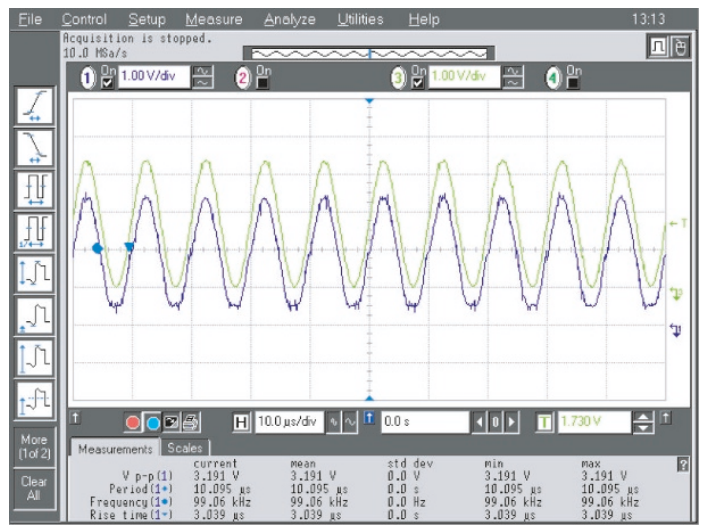

FIGURE 21: The comparison of original and captured $100 \mathrm{kHz}$ sine wave.

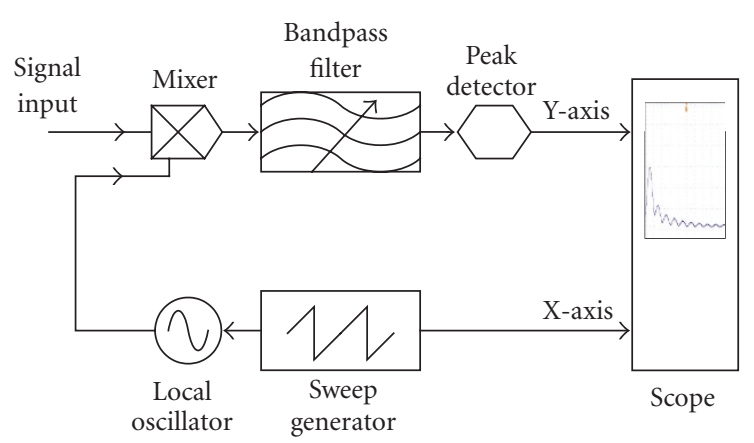

FIGURE 22: The function blocks diagram of real-time sweep spectrum analyzer.

where the changing frequency $f_{\text {in }}$ is the possible frequency component of the detected signal. 


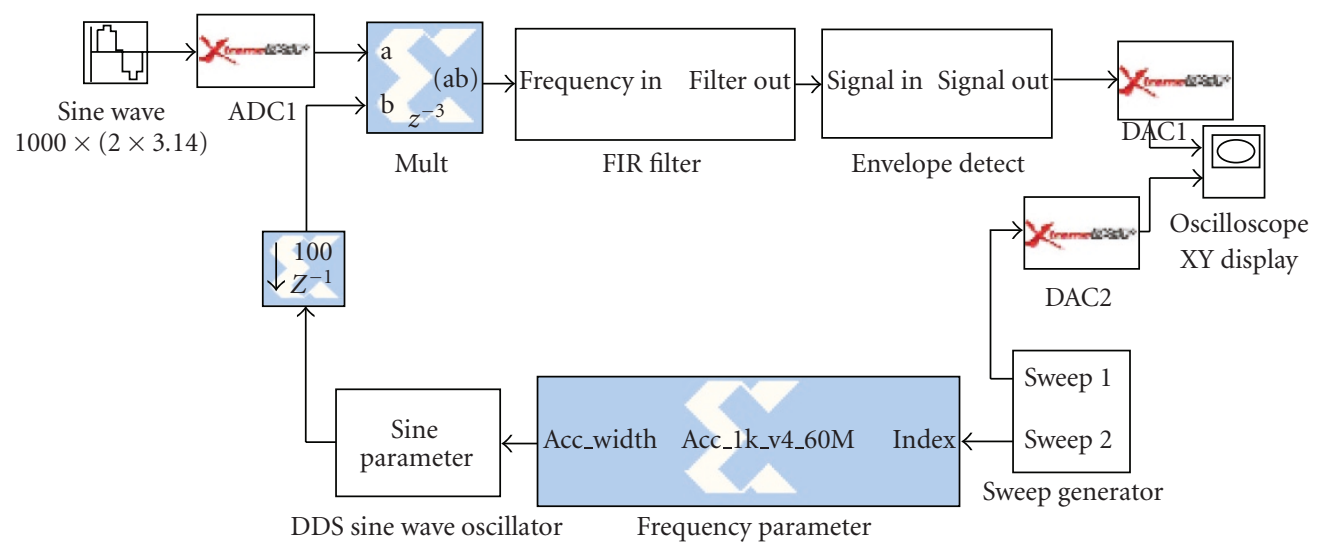

FIGURE 23: The signal processing flow of real-time sweep spectrum analyzer constructed by both Simulink and system generator.

TABLE 2: Analysis performance for the filters with different central frequencies.

\begin{tabular}{lccc}
\hline Central frequency & $1 \mathrm{MHz}$ & $100 \mathrm{kHz}$ & $1 \mathrm{kHz}$ \\
Frequency resolution & $10 \mathrm{kHz}$ & $1 \mathrm{kHz}$ & $10 \mathrm{~Hz}$ \\
& $10 \mathrm{kHz} \sim$ & $1 \mathrm{kHz} \sim$ & $10 \mathrm{~Hz} \sim$ \\
Scanning range & $2 \mathrm{MHz}$ & $200 \mathrm{kHz}$ & $2 \mathrm{kHz}$ \\
System clock rate & $60 \mathrm{MHz}$ & $60 \mathrm{MHz}$ & $60 \mathrm{MHz}$ \\
\hline
\end{tabular}

\subsection{FPGA realization}

We use software-hardware codesign and cosimulation approach to design a real-time sweep spectrum analyzer core, which can be built in a real-time electrical harmonic analyzer. First, we use the Simulink (with Matlab) function blocks to build up a sweep spectrum analyzer system, as shown in Figure 23.

In the same time, we integrate the Xilinx System Generator DSP Block Library [16] into the Simulink. With these library modules, we can cosimulate the total system and generate configuring bit for the corresponding FPGA chip so that we can perform the hardware verification. Software simulation can proceed by Simulink, or by ModelSim RTLsimulation. The Xilinx Chipscope is used to execute the hardware simulation and debugging.

Here, besides the ADC and DAC devices, we have designed an all-digital spectrum analyzer, where we use digital multiplier as the mixer, equiripple technique to implement FIR band-pass filter. The equiripple technique can make the attenuated band of frequency response of the filter equally smooth and optimizes the filter design.

\subsection{System performance}

We can implement multiple FIR filters in the same FPGA chip. Here, we demonstrate three different filters with central frequencies of $1 \mathrm{kHz}, 100 \mathrm{kHz}$, and $1 \mathrm{MHz}$, respectively. Table 2 shows their analysis performance.

The X-spacing is $20 \mathrm{~Hz}$ and the Y-axis represents the component amplitude in Figure 24. We notice that the odd harmonic peaks like $10 \mathrm{~Hz}, 30 \mathrm{~Hz}, 50 \mathrm{~Hz}$, and $70 \mathrm{~Hz}$ are clearly shown. The relative amplitudes ratios are the same as the expected by FFT calculation.

To verify the analysis resolution in the highest detectable frequency of $2 \mathrm{kHz}$ for the filter with central frequency of $1 \mathrm{kHz}$, we input an AM signal with carrier frequency of $2 \mathrm{kHz}$ and modulation frequency of $20 \mathrm{~Hz}$. Figure 25 shows the detected spectrum.

In Figure 25, the central frequency is $2000 \mathrm{~Hz}$, the left frequency is difference frequency of $1980 \mathrm{~Hz}$, and the right frequency is sum frequency of $2020 \mathrm{~Hz}$. The $X$-spacing is $20 \mathrm{~Hz}$. We can easily differentiate between different frequency components with resolution less than $20 \mathrm{~Hz}$.

The proposed digital IP can analyze the frequency span ranges from $10 \mathrm{~Hz}$ to $2 \mathrm{MHz}$. It can be flexibly utilized for FPGA-based real-time digital signal processing applications, such as visualized signal analysis, noise level monitoring, test and measurement of music studio, voice noise process, voice instruction interpreter, voice reading, and hearing aid design.

\section{FLEXIBLY RECONFIGURABLE SDI SYSTEM DESIGN}

The configuration of FPGA can be performed by either an on-line or an off-line process [17-19]. The dynamically reconfigurable SDI system is shown in the Figure 1. For the online process, we need a PC (personal computer) to connect with the instument for data exchange and on-line reconfiguring. The prestored configuration bitstream file for specified function can be used to reconfigure the FPGA via the controlling CPLD (complex programmable logic device). After powering up, the system will behave as a new instrument. The PC platform is able to perform the advanced analysis and $3 \mathrm{D}$ display of the data outputed from the instrument. 


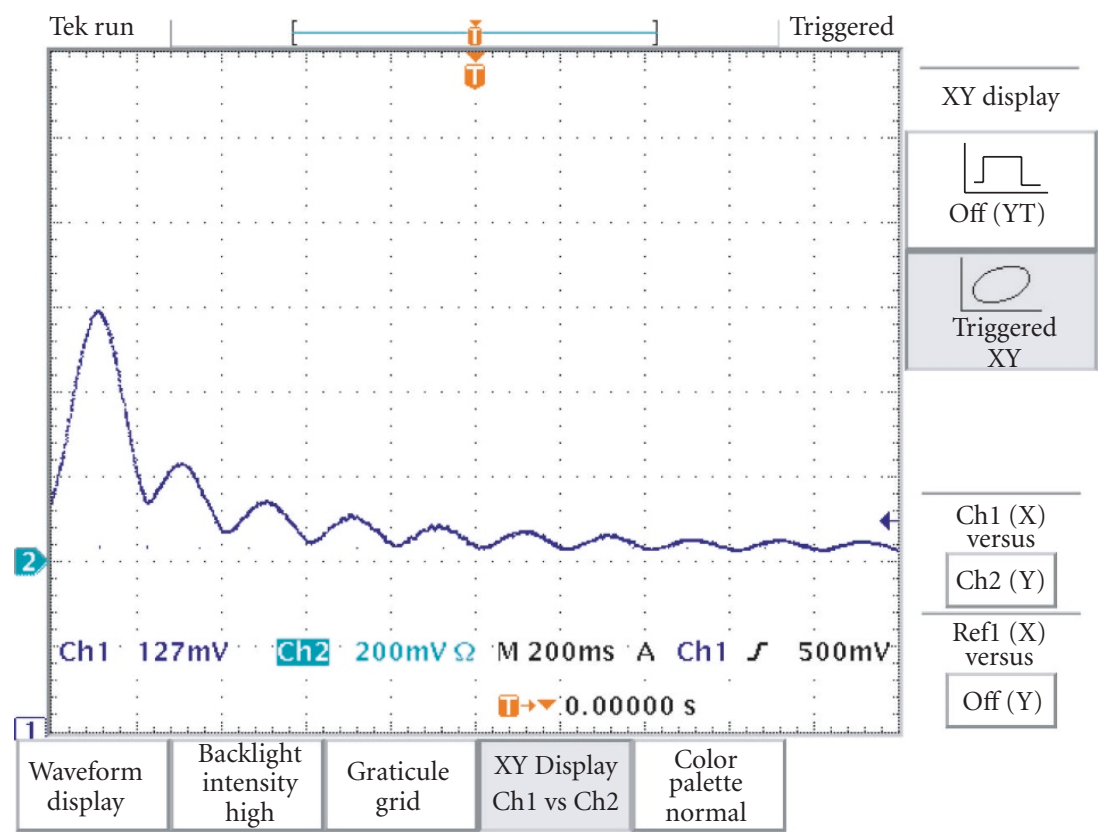

FIGURE 24: Analyzed spectrum of input square signal with period of $10 \mathrm{~Hz}$.

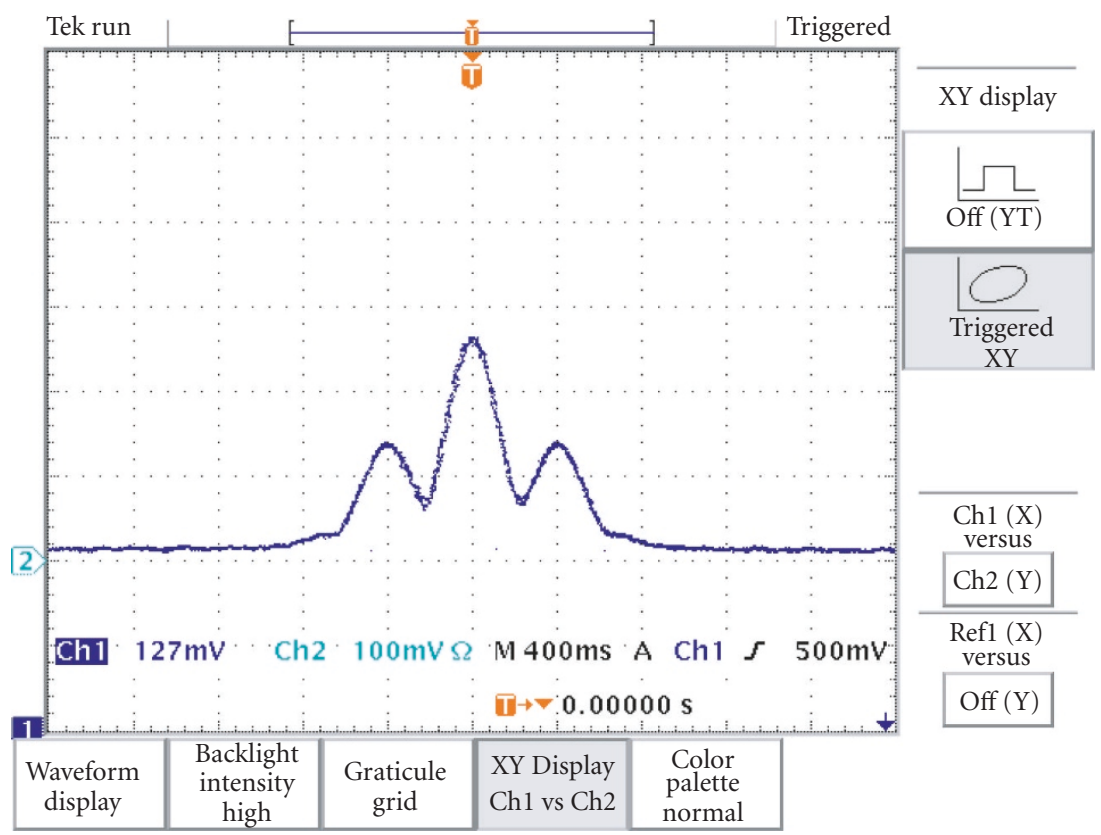

FIGURE 25: Detected spectrum of AM signal with carrier frequency of $2 \mathrm{kHz}$ and modulation frequency of $20 \mathrm{~Hz}$.

In another exchange way, the PC can also send more sophisticated excitation signals to the instrument for more specific applications. We can use LabVIEW programs or C codes to achieve these. For the off-line process, the functionpredefined configuring bitstream files are stored in different EPROMs (flash). We can use the function selection switch to order CPLD to send different CE (chip enable) signals to the selected configuring-EPROM (flash). When powered up, the instrument will be re-configured to work with new function.
Increasing or replacing different configuring EPROM (flash), we can add new functions to the proposed SDI system without changing or redesigning the system hardware.

\section{CONCLUSIONS}

The development trend of measurement and test technology is transferred from functionality-defined-by-manufacturer into functionality-defined-by-user. The ability of being 


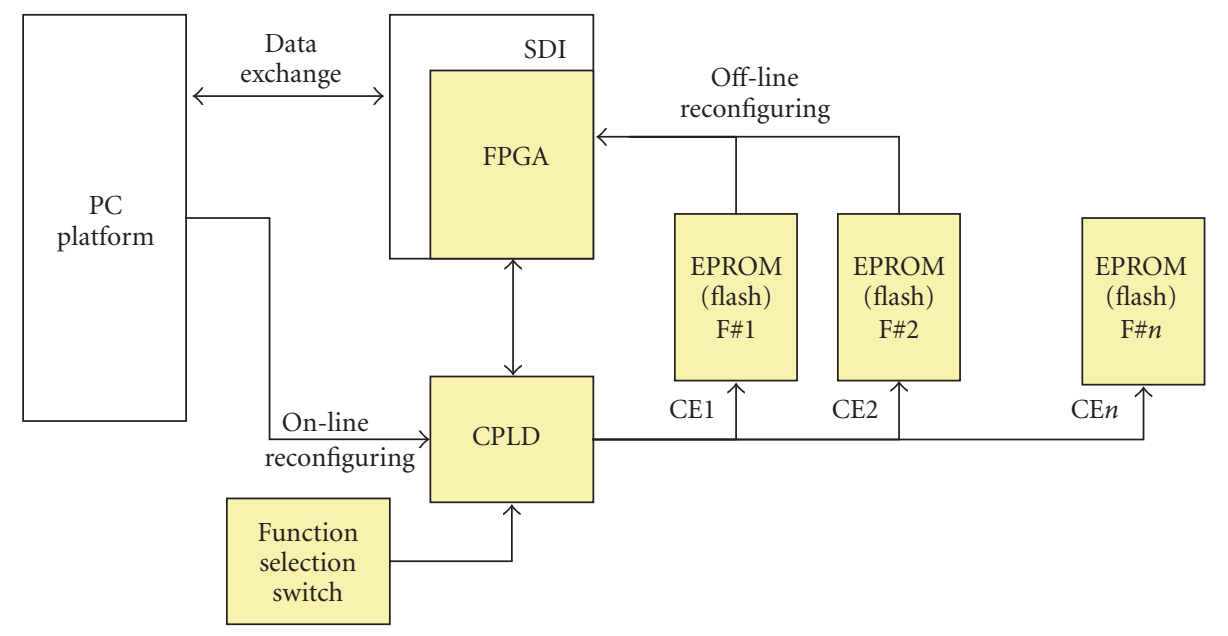

FIGURE 26: Flexibly reconfigurable SDI system.

reconfigurable, reusable, flexible, and rapidly prototyped will be the key to success in measurement and test market. This proposed FPGA-based reconfigurable instrument really meets the evolution trend. Once you have derived a measurement algorithm, you can easily build up a specialized instrument by SDI approach, such as an LCR (inductancecapacitance-resistance) meter, or biomedical monitor, and so forth.

\section{ACKNOWLEDGMENT}

This work was supported by National Science Council (NSC93-2215-E-168-004), Taiwan

\section{REFERENCES}

[1] Xilinx Incorporation, "The programmable logic data book," 2002.

[2] M. Cummings and S. Haruyama, "FPGA in the software radio," IEEE Communications Magazine, vol. 37, no. 2, pp. 108112, 1999.

[3] G.-R. Tsai, M.-C. Lin, G.-S. Sun, and Y.-S. Lin, "Single chip FPGA-based reconfigurable instruments," in Proceedings of International Conference on Reconfigurable Computing and FPGAs (ReConFig '04), Colima, Mexico, September 2004.

[4] S. A. Dyer, Survey of Instrumentation and Measurement, John Wiley \& Sons, New York, NY, USA, 2001.

[5] J.-W. Hsieh, G.-R. Tsai, and M.-C. Lin, "Using FPGA to implement a n-channel arbitrary wave form generator with various add-on functions," in Proceedings of 2nd IEEE International Conference on Field-Programmable Technology (FPT '03), pp. 296-298, University of Tokyo, Tokyo, Japan, December 2003.

[6] G.-R. Tsai, M.-C. Lin, W.-Z. Tung, K.-C. Chuang, and S.Y. Chan, "Wide-band and precisely measurement method of phase detector based on FPGA with embedded processor," in Proceedings of International Conference on Informatics, Cybernetics and Systems (ICICS '03), I-SHOU University, Kaohsiung, Taiwan, December 2003.
[7] G.-R. Tsai and M.-C. Lin, "High speed signal sampler by multiple-path algorithm," in Proceedings of IEEE Region 10 Conference (TENCON '04), vol. 1, pp. 29-31, Chiang Mai, Thailand, November 2004.

[8] G.-R. Tsai and M.-C. Lin, "Implementation of a real-time harmonic analyzer core by a single FPGA chip," in Proceedings of 25th Symposium on Electrical Power Engineering, Tainan, Taiwan, November 2004.

[9] J. Tierney, C. Rader, and B. Gold, "A digital frequency synthesizer," IEEE Transactions on Audio and Electroacoustics, vol. 19, no. 1, pp. 48-57, 1971.

[10] C. Dick and F. J. Harris, "Configurable logic for digital communications: some signal processing perspectives," IEEE Communications Magazine, vol. 37, no. 8, pp. 107-111, 1999.

[11] J. Vankka, M. Waltari, M. Kosunen, and K. A. I. Halonen, "A direct digital synthesizer with an on-chip D/A-converter," IEEE Journal of Solid-State Circuits, vol. 33, no. 2, pp. 218-237, 1998.

[12] W. C. Lindsey and C. M. Chie, "A survey of digital phaselocked loops," Proceedings of the IEEE, vol. 69, no. 4, pp. 410431, 1981.

[13] R. E. Best, Phase-Locked Loops: Design, Simulation, and Applications, McGraw-Hill, New York, NY, USA, 5th edition, 2003.

[14] T. Watanabe and S. Yamauchi, "An all-digital PLL for frequency multiplication by 4 to 1022 with seven-cycle lock time," IEEE Journal of Solid-State Circuits, vol. 38, no. 2, pp. 198-204, 2003.

[15] N. Kularatna, Modern Electronic Test and Measuring Instruments, IEE, London, UK, 1996.

[16] Xilinx System Generator v6.2 User Guide, 2004.

[17] Xilinx, "The Low-Cost, Efficient Serial Configuration of Spartan FPGAs," XAPP098, 1998.

[18] Xilinx, "Configuring Spartan-II FPGAs from Parallel EPROMs,” XAPP178, 1999.

[19] Xilinx, "Data Generation and Configuration for Spartan Series FPGAs," XAPP126, 2003. 
Guo-Ruey Tsai was born in Tainan, Taiwan, in 1953. He received the B.S. and M.S. degrees in electronics engineering from the National Chiao Tung University, Taiwan, in 1975 and 1977, respectively. Since 1993, he has been a Faculty Member at Kun Shan University, Taiwan, where he is currently an Associate Professor in the Department of Electronics Engineering. His research interests are in embedded processor architecture,

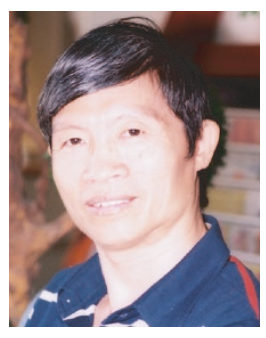
parallel processing algorithms, and FPGA-based instrumentation and measurement system design.

Min-Chuan Lin was born in Chang-Hua, Taiwan, in 1956. He received the B.S. degree from the National Taiwan University, Taiwan, in 1979, and the M.S. and Ph.D. degrees in electro-optical engineering from the National Chiao Tung University, Taiwan, in 1987 and 1992, respectively. Since 1993, he has been a Faculty Member at Kun Shan University, Taiwan, where he is currently an Associate Professor in the Depart-

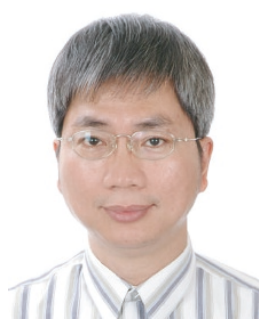
ment of Electronics Engineering. His research interests include FPGA-based reconfigurable system design and optical fiber transmission. 